\title{
What's New With JMR
}

This has been an exciting first quarter for Journal of Materials Research. We have begun to make improvements in four major areas, the first of which is affecting readers of JMR Abstracts in MRS Bulletin.

\section{Why JMR Abstracts are missing from this month's Bulletin}

The JMR production schedule has been shortened, beginning with the July issue. This means that accepted articles will appear faster in print. For the Bulletin reader, however, this means that JMR Abstracts will appear one month, rather than two months, prior to publication of the journal. Thus, in this April issue there are no June abstracts. They will appear in May. So this month and only this month, while the changeover is being made, JMR Abstracts are absent from the Bulletin.

\section{Full text of $J M R$ on the Web available to everyone for a limited time}

JMR Abstracts, as well as the full text of Rapid Communications, will continue to be seen on a regular basis on the MRS website prior to print publication. However, our second major area of improvement is the placement of the entire contents of $J M R$ on-line. For a trial period it will be available to everyone, while a permanent system for $J M R$ on-line access for subscribers only is developed. To view JMR on-line, visit MRS at www.mrs.org, then click on "JMR in its entirety." Visit now before it's only available to subscribers. Or better yet, subscribe to $J M R$.

Quicker review process for submitted articles

As with any peer-review journal, the speed at which JMR articles are reviewed can be dependent on the knowledgeable individuals who referee the submissions. Our third area of improvement is to tighten the review process by requesting that reviewers complete their evaluations on a reduced time frame. Our ultimate goal is to present quality papers quickly. By coupling a reduced review time with a new shorter production process, we intend to keep the same high quality archival journal, but make it more timely.

\section{Larger JMR issues published in 1999}

Our last area of improvement has already been apparent to readers of JMR Abstracts in the Bulletin, as well as to subscribers of JMR. The March, April and May issues of $J M R$ contained almost double the number of papers normally published. Other issues this year will also be slightly larger than normal. These larger editions are enabling us to eliminate the backlog of accepted articles. In the future this backlog reduction, coupled with the improvements mentioned above, will enable us to publish accepted materials more quickly.

\section{Ultimate Goal for JMR}

All of these improvements together should enable Journal of Materials Research to become an even more relevant publication for the new millennium, while maintaining its status as the premier archival journal in the materials research field.

Robert P. Frankenthal

Acting Editor-in-Chief

Journal of Materials Research 